\title{
La emergencia de las sociedades obesogénicas o de la obesidad como problema social
}

\author{
The emergence of obesogenic societies or \\ obesity as a social problem
}

Mabel GRACIA ARNAIZ

\section{RES U M E N}

En este artículo se muestra cómo la ideación biomédica de la obesidad y las propuestas preventivas que se han ido tejiendo institucionalmente en España durante la última década han contribuido a convertir el peso corporal y la comida en un problema social. La definición de los expertos acerca de las principales causas del incremento de la gordura ofrece información privilegiada sobre la concepción de las denominadas sociedades obesogénicas y sus estilos de vida, así como de las medidas adoptadas para cambiarlos, centradas principalmente en la responsablización individual y la educación nutricional. Este artículo demuestra que si bien hay una unanimidad en el diagnóstico, éste es insuficiente o no lo bastante preciso, ya que se debe saber más acerca de las consecuencias de los mudables modos de vida en las maneras de comer, y de éstas en la salud de los distintos grupos sociales. Un análisis de las iniciativas y programas de prevención elaborados en diferentes países sirve para dar cuenta de la estandarización de las estrategias internacionales frente a la obesidad epidémica y para reflexionar sobre los efectos de manejar una visión limitada de la cultura y la alimentación.

Palabras clave: Alimentación. Educación alimentaria y nutricional. Estilo de vida. Obesidad.

A B S T R A C T

This article shows how the biomedical conception of obesity and the preventive proposals that have been institutionally produced in Spain during the last decade have helped to turn the body weight and food into a social problem. The experts' definition relating to the main causes of increase in body fat provide paramount information on the concept of so-called obesogenic societies and their lifestyles. Normally, the means adopted to change lifestyles focus primarily on individual responsibility and nutritional educations. This article demonstrates that even though there is unanimity in the diagnosis, it is insufficient or not accurate enough, as it should enclose more detail about the consequences of changing eating habits, and how these influence the health of different social groups. An analysis of the initiatives and prevention programs developed in several countries may favor the standardization of international strategies against the obesity epidemic and serves to reflect on the effects of establishing a limited concept of culture and food.

Indexing terms: Feeding. Food and nutritional education. Life style. Obesity.

\footnotetext{
1 Universidad Rovira i Virgili, Departamento de Antropologia, Filosofia y Trabajo Social. Avda. Catalunya, 35 43002, Tarragona, España.
} 


\section{INTRODUCCIÓN}

Esta reflexión, estructurada en base a múltiples interrogantes algunos todavía sin responder, se enmarca dentro de una línea de estudios más amplia que tiene como objetivo analizar cómo y por qué determinados comportamientos alimentarios y prácticas corporales devienen problemáticos( ${ }^{(2)}$. Mediante un análisis de la obesidad desde fuera y desde dentro ${ }^{(3)}$, nos proponemos mostrar hasta qué punto el modelo biomédico de comprensión de la enfermedad y de intervención que se ha ido tejiendo institucionalmente en las sociedades contemporáneas y, en particular en España, ha contribuido en las últimas décadas a construir el peso y la comida en un problema social.

Desde hace unas décadas, los expertos en nutrición y dietética y las autoridades sanitarias destacan, una vez más, la importancia de la relación entre alimentación y salud. En la actual situación alimentaria, caracterizada por una relativa estabilidad y profusión de la oferta así como por una mayor accesibilidad, las recomendaciones adquieren un nuevo sentido al insistir ahora en que se debe comer menos, especialmente de ciertas sustancias (grasas y azúcares simples) y moverse más. Los consejos no sólo se circunscriben a qué alimentos hemos comer, sino que señalan cómo hay que vivir. Proliferan los estándares de buena alimentación y se advierte a la población de la necesidad de mantener una dieta prudente y equilibrada pues, con la abundancia derivada de la industrialización, los problemas de salud se han desplazado desde aquellos relacionados con la desnutrición durante la primera mitad del siglo XX, como el raquitismo, la pelagra o el bocio, hacia los relacionados con la sobrealimentación y el aumento de peso. Los profesionales de la sanidad hablan de una obesidad epidémica debida a un empeoramiento de los hábitos dietéticos producidos por un consumo excesivo de calorías y grasas y por el sobrepeso correspondiente que, en cuanto tal, es considerado un factor de riesgo para la salud. Se cuestiona, sin embargo, hasta qué punto este diagnóstico es acertado y lo suficientemente preciso.

Se parte de la hipótesis según la cual la obesidad se convierte en enfermedad cuando los expertos convienen que el exceso de peso no es sólo un efecto de gente que come demasiado o una cuestión de estética, sino que existe un vínculo de co-morbilidad entre éste y otras enfermedades crónicas, como la diabetes, la hipertensión o los problemas cardiovasculares. Las ideas que soportan la definición de la obesidad como una enfermedad crónica evitable llevan a los expertos en salud pública y las autoridades sanitarias a comprender y pensar su evolución en términos de una epidemia global - pandemia - y a identificar cada vez más los factores culturales como los principales causantes - el ambiente obesogénico - en detrimento, incluso, de las razones biopsicológicas. De esta forma, la obesidad se concibe como un problema de salud pública, con dimensiones morales y repercusiones sociales.

\footnotetext{
2 Con el soporte de programas de Investigación y Desarrollo del Ministerio de Educación y Ciencia (MEC), del Instituto de la Mujer y de la Generalitat de Catalunya, miembros del grupo de investigación del Departamento de Antropología, Filosofía y Trabajo Social (Universidad Rovira i Virgil) iniciaron en el año 2000 una línea de estudios sobre alimentación, género y salud. En 2006, con el apoyo de estas instituciones, la autora del presente artículo empezó un estudio sobre las dimensiones sociales de la obesidad en CIESAS (México, DF) y en la Université de Toulouse (Francia) que hoy continúa desarrollándose dentro de los proyectos La alimentación contemporánea desde y más allá de las normas (SEJ2006-15526-C02 02/SOCI) y iJóvenes gordos, pobres jóvenes?: procesos de discriminación y resistencia en torno a la obesidad (Ajove 2008). Esta reflexión parte, principalmente, del análisis de estrategias, guías alimentarias y discursos expertos sobre obesidad y recoge algunas consideraciones expuestas en publicaciones previas.

3 Se propone abordar la problemática de la obesidad desde "fuera" de la intervención asistencial y no desde las preocupaciones o intereses de los profesionales involucrados. Al mismo tiempo, se recurre al análisis desde "dentro" a través de la etnografía médica y, consecuentemente, del uso de herramientas cualitativas que favorezcan una aproximación holista y relacional. El trabajo se está desarrollando en tres niveles principales: a) revisión bibliográfica centrada en la literatura socioantropológica y epidemiológica, b) estudio de las recomendaciones nutricionales y estrategias sanitarias y c) etnografía médica realizada en Cataluña sobre representaciones y prácticas en torno a la obesidad.
} 
Se considera, sin embargo, que esta concepción favorece explicaciones sobre la causalidad cultural, a menudo, demasiado simples. Es el caso de mantener que en la medida en que los malos hábitos alimentarios se han globalizado, la obesidad se ha convertido en una enfermedad planetaria. Al entender, por otro lado, que su rápido y extraordinario incremento se ha producido en todo el mundo atendiendo a la misma razón, es decir, al consumo excesivo de calorías en relación a un menor gasto energético y a la sedentarización, ha justificado que las estrategias desarrolladas en los diferentes países con el objetivo de alcanzar hábitos de vida más saludables se homogenicen en contenidos y acciones. Así, pensar los actuales los estilos de vida como inadecuados y/o desestructurados está sirviendo, como se ilustra a continuación, para legitimar mecanismos de prevención e intervención en una dirección determinada - normativizar la vida cotidiana - y para reproducir y mantener ciertas prácticas biomédicas.

\section{Qué comer, cuánto pesar: la normativización dietética y corporal}

Los comportamientos alimentarios y los cuidados corporales han sufrido importantes cambios en relación a las sociedades tribales y épocas anteriores ${ }^{1}$. Al amparo de lo que Mennell ${ }^{2}$ ha llamado la civilización del apetito, en las sociedades modernas se ha ido popularizado, cada vez más, la vigilancia individual del peso corporal y la dieta en paralelo a la construcción de la delgadez corporal como un atributo de salud, de disciplina y de distinción social, de tal forma que estar delgado, aunque pueda relacionarse también con dolencias específicas, deja de ser un signo de enfermedad y pobreza para constituir un lugar de producción de nuevos significados. Contrariamente, la gordura pasa a representar un signo de trasgresión normativa y la consecuencia de aquello que no debe hacerse: comer mucho y ser ocioso.

Dichas concepciones hay que ubicarlas dentro de un largo proceso histórico de normalización dietética y corporal. Aunque esta normalización se remonta, al menos, a la antigüedad clásica toma renovada fuerza a partir del siglo XX cuando las autoridades sanitarias establecen, por un lado, un modelo nutricional basado en lo que se considera la dieta equilibrada u óptima -qué, cuánto, dónde, cómo, cuantas veces y con quién comer- $y$, por otro, un patrón de peso basado en el Índice de Masa Corporal (IMC) que, junto con otros indicadores de medición, define cuándo el volumen corporal es o no normal. La salud y la nutrición se convierten, así, en un factor esencial para la construcción de la ciudadanía, implicando cambios en las relaciones entre el estado, la sociedad y los individuos.

De acuerdo con Barona ${ }^{3}$, la nutrición deviene un elemento común de la cultura, la economía y la salud cuando el estado emerge como un regulador social y la producción y el consumo alimentario se entienden como una responsabilidad política. Durante esta centuria, los expertos en nutrición y el conocimiento científico adquieren una mayor atención para la mayoría de los gobiernos europeos, la sociedad civil y las organizaciones sociales y de beneficencia a consecuencia de los conflictos internacionales y de las crisis de los mercados. El hambre y la pobreza son consideradas un problema social y de salud pública y la provisión de alimentos se convierte en un derecho humano básico, envuelto de implicaciones morales: los hábitos alimentarios y la producción agrícola tradicionales tienen efectos negativos en la salud y la economía y, por el bien de todos, se han de modificar.

La noción de dieta optima, basada en la investigación fisiológica sobre la ingesta y el gasto energético y las necesidades de proteínas, grasas, minerales o vitaminas, se introduce en esta época, y significa el origen de la estandarización de los métodos usados en los estudios dietéticos, así como también la homogenización de los patrones alimentarios entre países y entre poblaciones rurales y urbanas: "el rol de los expertos nutricionales no sólo influye en el conocimiento, 
sino que también inspira la agricultura y las políticas sanitarias, la educación y las campañas preventivas con la intención de disciplinar y cambiar los hábitos populares" ${ }^{\prime 3}$. La regulación de la dieta y del peso es articulada, así, sobre la base de una responsabilización de los individuos sobre sí mismos, de una culpabilización frente a los otros y de la exigencia de una verdadera competencia nutricional $^{4}$. Se trata de un doble proceso, de medicalización y de moralización, según el cual hay que cambiar los malos alimentarios de la población y transformarlos en un nuevo conjunto de prácticas conformes a las reglas científicas de la nutrición que pretenden sustituir y/o condicionar las motivaciones económicas, simbólicas o rituales que condicionan los comportamientos alimentarios en cualquier cultura por otras de orden ditétetico y racional. Para motivar el seguimiento de prácticas más saludables se recurre a la idea de responsabilidad individual con un principal objetivo: comer de forma saludable no sólo produce satisfacción al paladar, sino que permite sentirse mejor con uno mismo (física y psíquicamente), con los demás (aceptación social) y con la sociedad (disciplina y control). Estos argumentos, que tan interesadamente han sido recogidos en los reclamos comerciales, están en la base de la concepción biomédica de la obesidad.

Por una parte, la medicina, durante decenios, ha prescrito a la población que adelgace, haciendo del régimen virtud. Por otra, cada vez más psiquiatras y nutricionistas condenan el culto excesivo de la delgadez corporal y advierten contra los efectos inversos de las dietas descontroladas en el aumento de peso. Los políticos proponen, a su vez, reglamentar las representaciones del cuerpo femenino en los medios de comunicación e intervenir, como ha sucedido en España, en la vigilancia del peso y el aspecto de las modelos de las pasarelas inventándose el concepto de belleza saludable ${ }^{(4)}$.
Sin embargo, ¿qué pueden hacer todas estas reglamentaciones políticas a favor del normo-peso frente al boyante mercado del cuidado del cuerpo? Éste es el que ha motivado profundos cambios culturales y es el combustible del que se alimentan numerosas profesiones y empresas. Los intereses de un sinfín de negocios están en la base de no pocos deseos por cambiar la apariencia física y mantener la calidad de vida y por ello es tan difícil discriminar en los discursos biomédicos qué hay de interés por la salud y qué por el lucro5. La asunción por la ciudadanía de los saberes biomédicos - hoy nadie discute las normas higiénicas - ha llevado a que el mercado utilice en su favor esos discursos expertos - prescindiendo de los profesionales o empleándolos como legitimadores-, para deconstruir y reconstruir permanentemente significados que alimentan la producción de bienes y servicios dirigidos al cuidado de uno mismo de todas las edades y géneros. Por eso existen poderosas razones en magnificar las cifras de la obesidad y convertirlo en un verdadero problema de salud pública y alimentar, con ello, el tan dilatado mercado de la salud. A las industrias farmacéuticas, alimentarias y a las empresas del body-building, al capitalismo de consumo en definitiva, les interesa la gordura igual que la delgadez: no dudan en animar la esbeltez a la vez que recriminan el sobrepeso, ni en alentar el ayuno a la vez que promocionan el hartazgo.

\section{Comer, engordar, enfermar: el diagnóstico}

En este contexto disonante, las autoridades sanitarias internacionales no dejan de insistir en que a los problemas de salud relacionados con la desnutrición se han sumado, en los últimos años, los derivados de la sobrealimentación y el sobrepeso. Definida como la acumulación excesiva

\footnotetext{
${ }^{4}$ Su contratación se limita a chicas con IMC entre 18-25 y se prohíbe maquillajes que den apariencia insana. Además, el Ministerio de Sanidad y Consumo ha firmado un acuerdo con empresarios y modistos para homogenizar las tallas. Esta acción, sustentada en un estudio antropométrico con 10415 mujeres entre 12 y 70 años, ha determinado tres morfotipos corporales para las españolas - diábolo (39\%), cilindro (36\%) y campana (25\%) -, estableciendo la tasa de obesidad en el $12.4 \%$.
} 
o anormal de grasa, la obesidad se describe como una enfermedad global, epidémica y multifactorial: afecta a todo el planeta, su prevalencia aumenta año tras año y en su origen no sólo están los factores genéticos o metabólicos sino los ambientales. Según la OMS más de 1600 personas adultas tienen sobrepeso $y$, de ellas, al menos 400 millones son obesas. La obesidad contribuye a incrementar las tasas de morbilidad y mortalidad al asociarse a enfermedades crónicas no transmisibles (ECNT) tales como la diabetes, la hipertensión o los problemas cardiovasculares ${ }^{(5)}$, incide en el aumento de muertes prematuras y la pérdida de años de vida saludable y eleva los costes asistenciales $^{6}$. Algunos la conciben, incluso, como una (socio) patología apoyándose en su desigual distribución, pues en las sociedades industrializadas y cada vez más en los países en transición o en desarrollo, afecta a las poblaciones con menos recursos socioeconómicos y, en según qué lugares y grupos de edad, más a las mujeres que a los hombres?.

Las cifras para Europa se consideran alarmantes, aunque son dispares ${ }^{(6)}$. En España, de acuerdo con la Encuesta Nacional de Salud ${ }^{8}$, el $37,8 \%$ de las personas de 18 y más años tiene sobrepeso y un $15,6 \%$ presenta obesidad. Entre la población de 2 a 17 años, el 18,7\% tiene sobrepeso y el $8,9 \%$ es obeso. Tanto en hombres como en mujeres, la obesidad es más frecuente a mayor edad, la prevalencia también ha crecido en Francia, aunque es menor. En 2003 la obesidad afectaba al $11,3 \%$ y en 2006 , con un aumento más limitado, al 12,4\%. Como en España, se presenta sobre todo entre los menores: el 16,0\% de los niños tienen ahora sobrepreso frente al $5,0 \%$ registrado en 1980 . Los hombres, en este caso, aventajan a las mujeres $-10,5 \%$ frente al
$8,4 \%-y$, de igual manera, la obesidad aumenta en función de la edad, con una sobrerepresentación en hombres a partir de los 36 años y en mujeres a partir de los 46 .

Aunque se aducen causas de origen endocrinológico, hipotalámico o genético, la obesidad más frecuente, la denominada exógena, habitualmente se explica por una ingesta energética superior a la necesaria. Sin embargo en España, según las estadísticas(7), la ingesta media de energía ha disminuido en más de 300 Kcal. en los últimos 50 años, aunque contrariamente el fenómeno de la obesidad parece crecer. ¿Cómo explicar esta aparente contradicción? En principio, la contradicción se resuelve apelando a dos diferentes tipos de causas, relacionadas entre sí: a) cambios profundos en la sociedad y b) cambios en el consumo alimentario. España, como otros países ${ }^{9}$, ha experimentado lo que se denomina una transición nutricional ${ }^{10}$, esto es, una secuencia de modificaciones en la alimentación, cuantitativas y cualitativas, relacionadas con transformaciones económicas, sociales, demográficas y con factores de salud.

Se dice que las dietas tradicionales han sido reemplazadas rápidamente por otras con una mayor densidad energética, lo que significa más grasas y más azúcar añadido en los alimentos, unido a una disminución de la ingesta de carbohidratos complejos y de fibra, de frutas, verduras y cereales. Estos cambios alimentarios se combinan con una reducción de la actividad física y, por tanto, del consumo energético, en el trabajo y durante el tiempo de ocio ${ }^{11}$. Algunos expertos han puesto de manifiesto la relación entre los dos tipos de transformaciones señaladas del siguiente modo: la comida "mala" es demasiado

\footnotetext{
5 Se ha establecido que la obesidad aumenta la probabilidad de aparición del síndrome metabólico, el cual hace regencia a un conglomerado de alternaciones que incluyen niveles elevados depresión arterial, glucemia y colesterol así como la acumulación de grasa en el tejido adiposo que, a su vez, incrementa el riesgo cardiovascular y de diabetes tipo 2.

${ }^{6}$ Hay que señalar que las cifras de prevalencia de la obesidad se manejan, a veces, con escasa prudencia. Lluís Serra advierte que la comunidad científica ha de tener en cuenta las diferencias metodológicas a la hora comparar ("Escenaris de Futur", Cosmocaixa, Barcelona, 4/05/2006).

7 En 1964, los Estudios Nacionales de Nutrición y Alimentación (ENNA-3 en Carbajal, 2005) indican que la ingesta media se situaba en torno a las $3008 \mathrm{Kcal}$. En la actualidad, tal como señala la Fundación Española de Nutrición, la media está en torno a las 2600 Kcal. En Cataluña, por su parte, la ingesta de energía es de 1981 Kcal. en 2002.
} 
fácil y barata como para no sucumbir a la tentación de encargar algo rápido y evitarse colas en los mercados, dinero y tiempo de preparación, lavado o cocción. "Se nos dice que debemos comer bien, pero al mismo tiempo las rutinas domésticas y dinámicas de trabajo actual se asientan en una comida rápida, fácil y barata"12.

Como se deduce de este tipo de consideraciones, los problemas de salud relacionados con la alimentación se explican cada vez más por la causalidad cultural, hasta tal punto que ha llevado a los expertos a calificar las sociedades contemporáneas de obesogénicas o entornos tóxicos ${ }^{13} \mathrm{y}$ a entender la obesidad como un fenómeno universal. Por esta razón, se afirma también que va a ser necesario un esfuerzo continuo durante muchos años, pues se trata de formar ciudadanos con una más clara capacidad de elección y además, en este caso, abordar las reformas estructurales y ecológicas en los territorios urbanos que faciliten una práctica generalizada de actividades físicas, en vez de convertirlas en una misión imposible: "Ilevará más de una década invertir esta tendencia... Las estrategias para afrontar el problema deben estar encaminadas a la adopción de hábitos alimentarios saludables y un estilo de vida activo por medido de la educación nutricional de la población y de la acción política que favorezca que la opción más saludable sea... accesible" 14 .

Este tipo de análisis es común en España y en países europeos próximos, así como en otros lugares, social y económicamente, más distantes. Así, por ejemplo, el diagnóstico establecido para Francia ${ }^{15} \mathrm{es}$, prácticamente, idéntico: la obesidad es una consecuencia directa de una alimentación excesivamente rica, sobre todo en lípidos, y gastos energéticos insuficientes. Por un lado, los comportamientos alimentarios anárquicos; por otro, una falta de ejercicio físico. La comida basura y el picoteo son los males de una época en la que, cada vez más, se recurre a la comida para resolver tensiones. Una alimentación industrial con platos ya preparados, grasas ocultas, una falta evidente de prótidos, que, sin embargo, podrían dar sensación de saciedad. Los productos destacados por la publicidad y su etiquetado, a menudo ilegible, falto de transparencia, cuando no engañoso (por ejemplo en el caso de los productos light o buenos para la salud). Los especialistas insisten, además, en las consecuencias del desarrollo del automóvil, de los transportes colectivos, en la generalización de la calefacción y el progreso de la industria textil, el aumento del tiempo pasado delante de la televisión o del ordenador y la disminución de los trabajos manuales.

Semejantes son las razones apuntadas para explicar el aumento de la obesidad en México, país donde las personas con sobrepeso y obesidad rozan el 69,9\% de la población según las cifras que manejadas por la Secretaria de Salud mexicana, considerándose uno de los lugares del mundo con las tasas de prevalencia más elevadas y ocupando el segundo lugar en el ranking después de EEUU: "[México] está experimentando una transición epidemiológica y nutricional que se refleja en una disminución lenta de los problemas asociados con el desarrollo,... al mismo tiempo que aumenta dramáticamente la prevalencia de la obesidad...y otras enfermedades crónicas no transmisibles, asociadas con la dieta y los estilos de vida"16. Las causas más citadas hacen referencia, como en los otros lugares, a las condiciones de vida actuales, tales como la creciente urbanización, el ritmo de vida acelerada, la desestructuración de la vida familiar, la mecanización de los medios de transporte, el sendetarismo, la inseguridad en las calles y la falta de equipamientos deportivos ${ }^{17}$.

En consecuencia, si el origen del problema parece ser el mismo en todas partes y tejerse en torno a esta cadena de causalidades sociales, ¿ por qué no han de serlo también las medidas para atajarlo?

\section{Controlar el peso, seguir la dieta, moverse más: las medidas}

Desde que el Índice de Masa Corporal (IMC) se ha establecido, no sin polémica ${ }^{18}$, en el 
indicador científico más utilizado para establecer el normo-peso o peso sano ${ }^{(8)}$, se está instruyendo a toda la población para que procure mantenerse entre sus límites porque hoy estar gordo se ha convertido en sinónimo de estar enfermo'. La obesidad no es sólo un problema de salud pública, sino económico(9): "combatir el problema de exceso de peso... contribuirá a reducir a largo plazo los costes para los servicios sanitarios... permitiendo a los ciudadanos tener una vida productiva hasta edad avanzada"19. Así, el argumento defendido por las autoridades sanitarias para atajarlo es triple4: es posible vivir mejor siguiendo una dieta equilibrada, se trabaja más si se goza de una buena salud y representa menos costos para la colectividad.

Este diagnóstico ha llevado a expertos y políticos de todo el planeta a elaborar múltiples propuestas para hacer frente a lo que se concibe como un fenómeno generalizado de mala alimentación y falta de ejercicio. La primera ha sido la Estrategia Mundial sobre Regimen Alimentario, Actividad Fisica y Salud (DPAS) adoptada por la 57a Asamblea Mundial de la Salud (AMS) en mayo de 2004 y desde entonces muchos países han emprendido acciones particulares.

Por ejemplo, en España se ha creado la Estrategia para la nutrición, actividad física y prevención de la obesidad ${ }^{20}$, con réplicas en numerosas comunidades autónomas ${ }^{(10)}$, en Francia se ha desarrollado el Programme National Nutrition Santé (PNNS 2006-10); y en México existe el Programa Nacional de Salud 2007-2012, que aunque nada más plantea impulsar una política intregral para prevenir y controlar la obesidad, no ha dejado de lanzar campañas y acciones específicas, tales como México está tomando medidas, Mídete la cintura, Por tu salud, ejercitate, Vive Saludable o Vamos a por el control. Por su parte, la UE tras afirmar que el aumento de la prevalencia de la obesidad afecta a toda Europa, ha elaborado, para apoyar y complementar las iniciativas nacionales, un marco de actuación común mediante la Plataforma Europea de Acción sobre Alimentación, Actividad Física y Salud ${ }^{19}$, la Red sobre Nutrición y Actividad Física y la Estrategia sobre Problemas de salud relacionados con la alimentación, el sobrepeso y la obesidad ${ }^{21}$.

No sólo sorprende la rapidez con la que las administraciones han respondido a la llamada de la $57^{a}$ de la AMS, sino la cantidad y la similitud de todas las acciones propuestas. Si bien la estrategia mundial ${ }^{22}$ precisaba que las decisiones sobre alimentación y nutrición estuvieran basadas en la cultura y las tradiciones y que, en consecuencia, los planes de acción nacionales tuvieran en cuenta estas diferencias, fueran culturalmente apropiados y se adecuaran a los cambios registrados en el curso del tiempo, lo cierto es que las primeros programas han sido extraordinariamente homogéneos y apenas han tenido en cuenta la diversidad sociocultural. En España, los programas integrales ideados por ciertas comunidades autónomas son casi idénticos, en parte porque se han adaptado a las directrices marcadas por la estrategia NAOS. Sin embargo, la prevalencia de la obesidad entre regiones es muy dispar. Mientras que en Asturias el 4.56 de la población entre 2 y 17 años es obesa, en Canarias la cifra se triplica, afectando al $15.88 \mathrm{de}$ niños y jóvenes. De forma paradójica, y a pesar de que las comunidades autónomas tienen competencias en sanidad, aquellas regiones que

\footnotetext{
8 El normo-peso o peso sano se ha establecido entre 18.5 y $24.9 \mathrm{~kg} / \mathrm{m}$. Algunos autores indican que hay cierta controversia sobre cuáles deben ser los puntos de corte a emplear para definir sobrepeso y la obesidad en la infancia y adolescencia. El IMC debe interpretarse, en cualquier caso, en el contexto específico de la edad y el sexo ${ }^{14}$. También se utiliza el cociente cintura/cadera, un indicador que mide la distribución de la acumulación adiposa (predominio superior o inferior) y que sirve para valorar el grado de riesgo metabólico o cardiovascular.

9 En Europa se estima que los costes directos e indirectos asociados a esta enfermedad suponen un $7 \%$ del gasto sanitario total ${ }^{21}$

10 Es el caso, por ejemplo, del Pla integral per a la Promoció de la salut mitjançant l'Activitat física i l'Alimentació Saludable (PAAS) en Cataluña, del Plan Integral de Obesidad Infantil en Andalucía 2007-2012 o del programa de Prevención de la Obesidad de la Consejería de Sanidad del Gobierno de Canarias.
} 
en primer lugar han emprendido iniciativas no coinciden, necesariamente, con las que presentan una mayor incidencia.

A su vez, las campañas institucionales de España, México y Francia hacen propuestas en una misma dirección: realizar de estrategias comunicación social y educación interactiva (webs específicas), crear observatorios de la alimentación, de la obesidad o de la calidad alimentaria, favorecer y promover la actividad física (espacios escolares, laborales, de ocio), mejorar el etiquetaje nutricional de los alimentos envasados, establecer alianzas estratégicas con todos los sectores implicados (por ejemplo, disminuir las raciones en la restauración, disminuir grasas y azúcares simples en los alimentos procesados), capacitar a los facultativos de primer nivel de atención, educar a padres y maestros, implicar a los medios de comunicación (mensajes anti-obesidad y proalimentación sana y actividad física en telenovelas, programas de gastronomía y divulgación) y, finalmente, movilizar a las colectividades territoriales/locales.

En lo relativo a los objetivos principales, estas estrategias refieren ítems donde, sin embargo, cuenta más la voluntad individual que el compromiso colectivo. Es el caso de animar a lograr un equilibrio energético y peso normal, a limitar la ingesta energética procedente de las grasas y azúcares o a hacer al menos 30 minutos de actividad regular, entre otros. Es evidente que es más sencillo enfazitar que los ciudadanos adquieran información nutricional que crear medidas que disminuyan las desigualdades sociales o cambien factores estructurales: " ¿cómo combatir la "concentración" de hábitos nocivos que suele darse con más frecuencia en determinados grupos socioeconómicos?"19. La respuesta de los políticos, siendo ilustrativa, poco tiene que ver con mejorar las condiciones de vida de los más afectados: "una educación adecuada de los consumidores constituye el primer paso para una elección con conocimiento de causa en materia de alimentación"19. De acuerdo con Arrestegui ${ }^{23}$, nos preguntamos sobre la eficacia de estrategias que ven la solución de todos los males en crear "consumidores mejor informados" y que, además, lo hacen a través de la adopción de normativas muy intervencionistas y de compleja aplicación(11). A la hora de reglamentar las declaraciones nutricionales y de propiedades saludables de los alimentos, a menudo solo incluyen consultas a las partes interesadas y acaban siendo reflejo del dogmatismo político de los diferentes grupos de presión (corporaciones empresariales, organizaciones de consumidores mejor representadas o grupos ecologistas). El legislador, animado por el consumerismo ilustrado, acaba adoptando normativas impracticables y con efectos contraproducentes: "todo para el consumidor, pero... jsin el consumidor!" 23 .

Junto a estas macroestrategias, proliferan las guías de buena alimentación que tratan contrarrestar los efectos del denominado ambiente obesogénico. Se advierte a la población de que, para evitar la obesidad, hay que prescindir de las motivaciones no-racionales que guían las preferencias alimentarias, porque una vez instaurada se convierte en una enfermedad de difícil tratamiento: "cuando no nos sentimos bien lo compensamos comiendo y bebiendo, aunque no tengamos apetito y en contra de nuestro raciocinio y nuestra salud" 20 . A la hora de prescribir dietas de adelgazamiento, numerosos facultativos consideran que los portadores de la anomalía -el exceso de grasa- son responsables de su disfunción', si usted está obeso es porque come mucho o porque no sabe o no quiere comer bien. En el caso de que las personas gordas sean niños, esta responsabilidad se traslada con facilidad a los progenitores, como sucedió en 2007 en Gran Bretaña, cuando la madre de Connor McCreaddie, un niño de 9 años y 89 kilos de peso, estuvo a punto de perder la custodia de su hijo acusada de

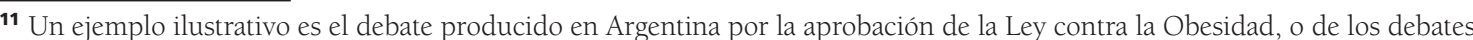
que en los parlamentos europeos se están produciendo para legislar sobre este ámbito. El actual gobierno español pretende tramitar en la actual legislatura las leyes de Salud Pública y Seguridad Alimentaria.
} 
negligencia o del niño asturiano que, por lo mismo, lleva apartado de su familia desde junio del 2006. Custodiado por los servicios sociales de Asturias ha logrado perder 40 kilos de los 100 que pesaba, y no va a regresar a su hogar hasta que "el ambiente familiar propicie los hábitos saludables del pequeño".

Si bien los programas de prevención están basados en acciones comunicativas positivas y dicen alejarse de las teorías que culpabilizan y responsabilizan al individuo de su enfermedad definiendo el problema de la obesidad como un mal de carácter social y colectivo, lo cierto es que el trasfondo ideológico de las intervenciones no es otro que el de recordar que, al fin y al cabo, estar gordo sólo depende de uno mismo: "la persona es en última instancia responsable de su estilo de vida y del de sus hijos"21. Al concebir los comportamientos alimentarios de la mayoría de la población como problemáticos, los consejos facultativos se convierten en la vía para regularlos durante todo el ciclo vital y la educación nutricional en el baluarte moral de los estilos de vida saludables. Todas las guías alimentarias señalan en la misma dirección. En tanto que los "hábitos se inician a los tres o cuatro años y se establecen a partir de los once, con una tendencia a consolidarse a lo largo de toda la vida", el control sobre la comida se debe ejercer en edades tempranas y desde la primera ingesta del día: "la familia, como primera transmisora de mensajes, debe tener unos conocimientos básicos sobre alimentación saludable"20. Se pretende, en definitiva, que el individuo sano o enfermo modifique su conducta por el convencimiento de que hay una racionalidad científica que le puede ayudar: "sólo un consumidor bien informado puede adoptar decisiones razonadas"21. Se comprende entonces que las personas más pobres sean más obesas porque, siguiendo esta lógica cientifista, tienen menos acceso a la información: "... un nivel bajo de educación y un acceso más limitado a la información reducen la capacidad para elegir con conocimiento de causa" ${ }^{19}$.

Ahora bien, ¿hasta qué punto, por muy racionales que sean estas medidas están siendo eficaces? No deja de ser sorprendente que, supuestamente, la tasa de obesidad haya aumentado ${ }^{19}$ coincidiendo con el esfuerzo educativo que las autoridades sanitarias han emprendido desde hace décadas para enseñar hábitos de vida saludable y con el hecho que la población muestre un conocimiento óptimo de las recomendaciones nutricionales. ¿Dónde está, entonces, la utilidad de estas múltiples (y costosas) acciones? ¿Cómo se están llevando a cabo? ¿Se trata de acciones coordinadas con cobertura en todo el territorio estatal o, de momento, son medidas especificas, con poca interrelación entre sí y de incidencia territorial muy variable? Es más ¿cómo se está evaluando su impacto? Apenas, hay datos disponibles sobre los efectos de las iniciativas emprendidas y las autoridades sanitarias advierten que tampoco podrán ser evaluadas a corto plazo(12).

En Francia, sin embargo, se han atribuido ya ciertos logros a las campañas institucionales(13). La actual estrategia francesa PNNS, ya en su segunda fase (2006-2010), presenta novedades significativas respecto a la anterior. Parte de diez principios generales, entre los que destaca que la elección alimentaria es un acto libre, en el contexto cultural y social propio de cada persona, y que hay que vigilar los mensajes difundidos y no focalizar las acciones de prevencion sobre la obesidad, que es lo mismo que decir hay que desmedicalizar los mensajes de prevención. Esta segunda versión, en la que han participado

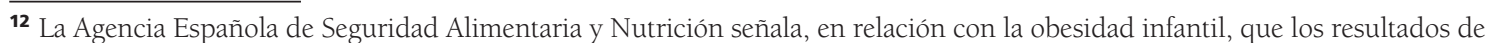
la estrategia NAOS no podrán conocerse antes de diez años

13 En los dos últimos meses, la prensa escrita ha hecho público algunos estudios relativos a Francia y también EEUU señalando que, por primera vez desde 1980, la tasa de obesidad infantil se ha estancado. Los investigadores no saben, sin embargo, si estamos ante una casualidad estadística o una tendencia a largo plazo, aunque, eso sí, todos coinciden en afirmar que el éxito hay que atribuirlo a las políticas gubernamentales (disponible en http://www.nlm.nih.gov/medlineplus/spanish/news/ fullstory_64648.html; www.lavozdegalicia.es/sociedad/2008/05/28/00031211967335352732582.htm).
} 
también expertos de ciencias sociales, trata de enmendar algunos de los problemas considerados de base, tales como insistir en recomendaciones nutricionales conocidas por todos, generalizar causas y abordajes y estigmatizar la obesidad. El PNNS toma en cuenta la triple dimensión biológica, simbólica y social del acto alimentario y destaca una serie más amplia de medidas concernientes a poblaciones específicas. Dicha especificidad se concreta en hacer intervenciones diferenciadas según diferentes edades de la vida, objetivar las acciones sobre las poblaciones desfavorecidas a partir de tener en cuenta las redes sociales en las acciones de educación para la salud y mejorar la ayuda alimentaria aportada a las personas en situación de precariedad.

Parte de estas ideas han sido incorporadas en el Second WHO European Action Plan for Food and Nutrition Policy 2007-2012 (septiembre, 2007) destacando, una vez más, la necesidad de adaptar los programas según cada contexto. Habrá que ver, en adelante, cómo se despliegan y qué consecuencias tienen en la práctica, dado que estos argumentos ya fueron recogidos, en parte, en la 57ª Asamblea Mundial de la Salud en 2004 sin mucho éxito.

\section{Una concepción limitada de la cultura y la alimentación: la discusión}

El diagnóstico presentado en los apartados anteriores alude a profundos cambios en los estilos de vida y las prácticas alimentarias. Para los gestores de las políticas sanitarias se está ante una enfermedad transnacional que podría evitarse, en buena medida, aprendiendo a comer bien y aumentando la actividad física. El diagnóstico parecería correcto si la obesidad fuera un fenómeno reciente y generalizado, pero es incierto o no lo suficientemente preciso si se relativiza su carácter global y se matizan ciertas relaciones de causa-efecto entre gordura y enfermedad.
En este sentido, faltan reflexiones a diferentes niveles. Quizá la más relevante tenga que ver con la necesidad de pensar la alimentación como un fenómeno complejo que engloba aspectos biológicos, psicológicos y sociales $y$, en consecuencia, establecer un diálogo pluridisiciplinar entre las ciencias sociales y las ciencias de la salud ${ }^{24}$. Del mismo modo que con cierta frecuencia la sociología o la antropología han explicado las prácticas alimentarias determinadas por factores exclusivamente sociales, en la biomedicina existe una visión fragmentada del ser humano y la cultura con efectos muy particulares en su comprensión de los problemas.

Este texto no cuestiona, en principio, los diversos porcentajes de prevalencia de obesidad presentados por los estudios epidemiológicos ni tampoco la multitud de modelos explicativos para atajarla. Lo han hecho los mismos expertos en salud pública ${ }^{(14)}$. Parece prudente, sin embargo, mantener una cierta distancia con las cifras relativas a su incidencia dada la diversidad teóricometodológica de las investigaciones y el consecuente baile de números entre unas y otras. Parece oportuno, también, preguntarse si la obesidad es, ciertamente, un fenómeno nuevo o, si acaso, ya existía en las sociedades en una proporción superior a la que ahora se apunta para defender su carácter epidémico.

Hoy los datos epidemiológicos sobre el aumento del sobrepeso y la obesidad son leídos por los expertos sanitarios como síntomas de que la sociedad industrializada va mal ${ }^{25}$, asimilándose como evidente que la grasa mata, que la gordura es en sí misma patológica y que todos los obesos son o serán forzosamente enfermos ${ }^{26}$. En esta línea, se señala de forma recurrente que es más probable que los niños obesos sean adultos obesos que los niños que no lo son, aunque, como indican ciertos estudios, la mayor parte de los adultos obesos en la actualidad no fueron niños obesos ${ }^{14}$. Tampoco hay consenso científico sobre si, a la

\footnotetext{
${ }^{14}$ Las explicaciones sobre el fenómeno de la obesidad, y las propuestas de abordaje, varían dependiendo de los modelos utilizados, ya sean desde la epidemiología sociocultural y/o clásica o ambas a la vez. En los programas de atención y prevención predominan cada vez más los enfoques eclécticos (Socorro Parra-Cabrera et al, 1999) y los enfoques denominados ecológicos. Para una revisión crítica de los actuales modelos explicativos véase Lang y Rayner ${ }^{29}$.
} 
hora de establecer relaciones entre peso corporal y mortalidad, conviene más estar por encima del normo-peso que por debajo y no son pocos los estudios que consideran más efectivo el ejercicio físico que la dieta(15). Entonces ¿por qué cuando se manejan las cifras sobre obesidad se tiende a sumar los porcentajes del sobrepeso en lugar de discriminarlos? No se argumenta que sólo ciertos grados de gordura y en cierto tipo de personas pueden relacionarse con el aumento de la morbomortalidad y que, en esos casos, ponerse a dieta no siempre es una solución.

Por otro lado, con frecuencia se considera que los problemas alimentarios, y en particular aquellos relacionados con el peso tienen su origen en la cantidad de comida ingerida, como si los efectos de comer en el cuerpo fueran una simple operación aritmética: se come poco o demasiado y se engorda o adelgaza según las calorías consumidas/gastadas. La realidad es menos simple, ya que el peso dista de depender exclusivamente de las cantidades ingeridas de alimentos. Intervienen, junto con el ambiente, mecanismos hormonales y neurales, factores genéticos, metabólicos y constitucionales ${ }^{27}$ que no hay que menospreciar. El modelo epidemiológico clásico acepta la cadena de causalidades y reconoce que la obesidad no es provocada por una única razón: "sabemos que la obesidad se produce cuando coinciden en una misma persona la predisposición genética y los factores ambientales desencadenantes"7. De hecho, la obesidad mórbida o extrema - aquella que de forma más evidente parece incidir en el aumento de ECNT - responde, en numerosas personas, a alteraciones fisiológicas $y$, frente a éstas, seguir una dieta restrictiva no resuelve, necesariamente, el problema.

Tampoco parece oportuno relacionar el aumento de la obesidad sólo con la profusión alimentaria característica de las sociedades modernas y los malos hábitos adquiridos ante la abundancia. ¿Es cierto que, como señalan políticos y expertos, los consumidores modernos no saben comer? ¿Es verdad que los comportamientos alimentarios están cada vez más desestructurados? Diversos estudios señalan que la desestructuración alimentaria en las sociedades modernas es solo relativa ${ }^{24}$, ya que si bien se ha producido una simplificación e individualización de las comidas, así como un ligero aumento del numero ingestas diarias, no es posible establecer una relación directa entre dichas tendencias y la obesidad. Aceptar la premisa de la desestructuración alimentaria y sus efectos negativos en la población puede ser útil para legitimar acciones en educación nutricional, pero no es científicamente sostenible asociarla a un empeoramiento de la salud. Si la paulatina degradación del orden social y alimentario fuera cierta, entonces también se haría difícil afirmar, como se ha hecho, que el incremento de la esperanza de vida experimentado en las últimas décadas se haya debido, en parte, a una mejor alimentación.

En cualquier caso, y ante tanta controversia, parece evidente que la actual concepción de la gordura como enfermedad no sólo está contribuyendo a aumentar el pánico - físico y moralfrente a las grasas y el sobrepeso, sino a estigmatizar aún más a las personas obesas. No es de extrañar, que en este contexto generalizado de rechazo hayan aparecido grupos de activistas y científicos que reclaman la aceptación de la gordura ${ }^{28}$.

Referirse al entorno (obesogénico o lipófobo) cuando se trata de buscar las causalidades y/o responsabilidades de ciertos problemas de salud significa no definirlo como una especie de nebulosa abstracta y compleja (y por tanto difícilmente abordable), sino aprehenderlo

\footnotetext{
$\mathbf{1 5}$ Mientras que a nivel de estudios clínicos parece haber unanimidad en que establecer relaciones entre obesidad (IMC mayor o igual a 30) y aumento de comorbilidad, no la hay con respecto a sobrepeso y otras ECNT. Lo mismo sucede con el debate suscitado por Katherine Flegal, según la cual los estudios de base estadística atribuyen, para poblaciones amplias en EEUU, un exceso de muertes asociadas a la obesidad, aumentado incesariamente la alarma sobre el sobrepeso. Cabe citar también que la bibliografía biomédica señala que perder peso en exceso aumenta la mortalidad en personas con sobrepeso y obesidad, que las personas que presentan oscilaciones continuas de peso (subidas y bajadas) presentan una mayor mortalidad que las que, aun teniendo sobrepeso u obesidad, mantienen el peso estable a lo largo de la vida ${ }^{27}$. Por su parte, Gerardo Villa, profesor de la Universidad de León, apunta que la escasa actividad física, más que la mala alimentación, es la causa de la obesidad infantil.
} 
en tanto que organización misma de una sociedad y en tanto que fruto de procesos históricos dinámicos y de amplio alcance ${ }^{1}$. A pesar de la creciente globalización, la obesidad no afecta de igual modo, en todo el mundo. Ni todos los gordos están enfermos ni todos comemos mal. Su incidencia es muy desigual atendiendo a diferencias intra e interculturales. Lo hemos visto para España, Francia o México. En estas sociedades, el nivel socioeconómico, el género, la edad o el origen étnico constituyen variables explicativas. Y no sólo porque las oportunidades de alimentarse y de gestionar la salud son muy distintas según dichas variables, sino porque las prácticas alimentarias dependen de otros factores micro y macroestructurales.

¿Por qué para combatir la obesidad no se bajan los precios de los alimentos saludables, se acaba con la comida "basura" o se aumenta las oportunidades de los más pobres? ¿Acaso no son más perniciosos para la salud las largas jornadas trabajo mal remuneradas o el hiperconsumo de actividades tan variadas como dispares? Poco sabemos sobre la incidencia de estas prácticas en la obesidad. Sí sabemos, en cualquier caso, que las actuales estrategias no han sido diseñadas para enfrentarse a estas u otras cuestiones sociales. Al contrario, buena parte de los programas de prevención se estructuran sobre una concepción simple de la cultura y los estilos de vida, según la cual si se consigue cambiarlos mediante la adecuación de las conductas individuales se logrará combatir la obesidad y otras enfermedades asociadas. Los factores socioculturales se entienden sólo como agentes específicos causantes de enfermedad/muerte que pueden ser abordados aisladamente.

Lang y Rayner ${ }^{29}$ proponen que ha llegado el momento de repensar la salud pública evitando, en primer lugar, la cacofonía política derivada de la multitud de modelos explicativos de la obesidad y proponiendo, a continuación, una aproximación alternativa que incida menos en las respuestas soft, del tipo hay que comer más o menos, modificar etiquetaje de los alimentos o educar en nutrición, y más en las respuestas hard; es decir, relacionando los ámbitos físico, fisiológico, social y cognitivo que, de diferentes maneras y grados, están en la base de los procesos de salud/ enfermedad. En este sentido, es evidente que nuestros comportamientos, incluido el alimentario, tienen un componente claramente estructural y aunque, como señala Luque $^{30}$, es cierto que los individuos tienen capacidades para reapropiarse del sentido de sus actividades, hay prácticas que dependen de y están en relación con lógicas económicas y políticas más amplias que hay que atender.

\section{CONCLUSIONES}

Las autoridades sanitarias españolas han declarado la guerra a la obesidad advirtiendo a todos que sobrepasar el peso normal nos convierte en futuras víctimas de la hipertensión, la diabetes o el infarto. Promocionan acciones contra el peso excesivo o las dietas milagro, crean webs para difundir información sobre las nefastas consecuencias de seguir regímenes poco rigurosos, ofrecen consejos sobre la forma saludable de perder peso e invitan a las industrias a reducir las grasas y azúcares de sus productos. No son pocas las propuestas para legislar en este ámbito, como ya se ha hecho en otros países. Es así como los mensajes anti-obesidad inundan los medios de comunicación, las consultas médicas, las escuelas, las oficinas. Sin embargo, es necesario preguntarse hasta qué punto esta extraordinaria problematización del peso y la comida está contribuyendo a frenar la obesidad o, por el contrario, animándola, haciendo de la dieta, no una acción, sino un estado. Ponerse a dieta ha sido y sigue siendo recomendado sistemáticamente por muchos clínicos al margen, incluso, de que se haya podido demostrar que el dieting está en la base de no pocas obesidades.

En las estrategias integrales y en los modelos explicativos de la obesidad faltan enfoques interdisciplinares que contribuyan a una mejor comprensión teórica y práctica de este problema y, en particular, de su naturaleza y dimensiones sociales. Faltan, en definitiva, propuestas reflexivas que asuman la historicidad 
de los procesos que lo atraviesan y que articulen los diferentes niveles implicados. En este sentido, urge también reformular el uso de la causalidad social a la hora de explicar ciertas tendencias. Es relativamente fácil establecer una batería de posibles causas socioculturales en el origen y evolución de la obesidad; sin embargo, es más difícil demostrarlas. Se han tipificado como agentes causales desde la alimentación industrializada al ocio pasivo, desde la desestructuración familiar al ritmo acelerado de vida, desde la generalización de la calefacción o el transporte mecanizado a la falta de equipamientos deportivos. Ahora bien ¿cómo se está sustentado empíricamente la correspondencia entre tantos - y tan dispares- factores y el incremento de personas obesas? Se conviene, por ejemplo, que el aumento sedentarismo es uno de ello, pero ¿hay estudios longitudinales que muestren desde cuándo y en qué medida los españoles hacemos menos ejercicio ahora que hace treinta años?

Aún conviniendo que fuera cierta la rápida progresión de la obesidad y la causalidad cultural su principal factor explicativo, hay que reconocer que las acciones emprendidas hasta la fecha se han mostrado, cuando menos, poco eficaces. Quizá la razón de ello se deba, en parte, al manejo de excesivas suposiciones. Antes de pretender modificar la alimentación, hay que saber más acerca de las causas y consecuencias de los mudables modos de vida en las maneras de comer, y de éstas en la salud de los distintos grupos sociales. Las prácticas que, no sólo en apariencia, sean nocivas para la salud se han de tomar, también, como aspectos de la vida cultural y condicionadas por factores socioculturales. Para ello es imprescindible trabajar con una concepción menos limitada de la cultura y la alimentación.

Mejorar los hábitos alimentarios no es una tarea fácil a pesar del empeño y de los medios que puedan desplegar las administraciones o instituciones más o menos responsables. Hasta hoy se ha puesto el acento, casi exclusivamente, en la educación nutricional. Y la educación nutricional se ha entendido, y sigue entendiéndose, fundamentalmente, como proporcionar información orientada a lograr una dieta equilibrada. Pero no es así, ya que la certeza científica no es una razón suficiente para asegurar el éxito de las recomendaciones de los expertos. Estudios del Observatorio de la Alimentación ${ }^{31}$ han puesto de manifiesto en España que, por un lado, la población está satisfecha con su alimentación ya que considera que es buena y sana al mismo tiempo y, por otro, que la gente reproduce las recomendaciones de los expertos en relación a lo que cabe considerar una alimentación saludable y equilibrada. Las normas interiorizadas por la mayoría de la población española ponen de manifiesto, en esta línea, un buen nivel de apropiación de los discursos nutricionales. A pesar de ello, las prácticas alimentarias siguen motivadas y condicionadas por diferentes constreñimientos materiales y simbólicos, de tal forma que no parece existir una correspondencia directa entre las recomendaciones dietéticas asumidas por las personas y los consumos realizados.

Existen, por otro lado, serias dificultades para implantar, en una cotidianidad pautada por imperativos múltiples, la rutina que recomiendan los consejos dietéticos. Las exigencias diarias de muchas personas no permiten un régimen nutricional más saludable, más equilibrado y más conveniente para su salud, al menos en la medida que quisieran las autoridades sanitarias, porque para cambiar de dieta es necesario cambiar de vida, lo cual, como han mostrado estudios etnográficos y sociológicos previos, no sólo es siempre difícil sino que puede llegar a ser, para determinadas personas, imposible. El diagnóstico que se ha sintetizado aquí insiste más en los productos consumidos que en las actitudes o razones por las que unos alimentos son o no ingeridos. Y, así, si las medidas se orientan a modificar los alimentos o las actitudes individuales en lugar las razones estructurales que dan lugar a los desequilibrios alimentarios ¿no se estará desatinando en las repuestas o retrasando las soluciones? Ignorar estas relaciones y especificidades es, casi con seguridad, condenar al fracaso cualquier estrategia preventiva. 


\section{REFERENCIAS}

1. Gracia M. Comer bien, comer mal: la medicalización de la alimentación. Salud Pública México. 2007; 49(3):236-42.

2. Mennell S. All manners of food. Eating and taste in England and France from the Middle Ages to the present. London: Basil Blackwel; 1985.

3. Barona JL. Nutrition and health. The international context during inter-war crisis. Social History Med. 2008; 21(1):87-105.

4. Ascher F. Le mangeur hypermoderne. París: Odile Jacob; 2005.

5. Gracia M, Comelles JM, editores. No comerás. Barcelona; 2007.

6. Le Guen JM. Obesité, le nouveau mal français. París: Armand Colin; 2005.

7. Barbany M, Foz M. Obesidad: concepto, clasificación y diagnóstico. An Sistema Sanitario Navarra. 2002; 25 Supl 1.

8. Encuesta Nacional de Salud. Ministerio de Sanidad y Consumo. 2006. Disponible en: <http://www. msc.es/estadEstudios/estadisticas/encuesta Nacional/encuestalndice2006.htm>.

9. Lambert JL, Batalha MO, Sprolsser RL, Silva AL, Lucchese T. As principais evoluções dos comportamentos alimentares: o caso da França. Rev Nutr. 2005; 18(5):577-91.

10. Popkin BM. Nutritional patterns and transitions. Population Dev Rev. 1993; 19(1):138-57.

11. Chavarrias M. La obesidad se asocia a los nuevos hábitos alimentarios. 2005. Disponible en: <http:// www.consumaseguridad.com>.

12. Montaner J. El reto de aprender a comer. 2004. Disponible en: <www.consumaseguridad.com>.

13. Henderrson KE, Brownell KD. The toxic environment and obesity: contributions and cure. In: Thompson JK, editor. Handbook of eating disorders and obesity. New York: Wiley and Sons; 2004.

14. Aranceta J. Obesidad infantil: nuevos hábitos alimentarios y nuevos riesgos para la salud. In: Díaz C, Gómez C. Alimentación, consumo y salud. Barcelona: Fundació "La Caixa; 2008.

15. Basdevant A, Guy-Grand B. Médecine de l'obésité. París: Flammarion; 2004.

16. Barquera $S$, et al. Sobrepeso y obesidad. México: Instituto Nacional de Salud Pública; 2006.

17. Fausto GJ, et al. Antecedentes históricos sociales de la obesidad en México. Inv Salud. 2006; 8(2) 91-4.
18. Rubio MA, et al. Consenso SEEDO 2007 para la evaluación del sobrepeso y la obesidad y el establecimiento de criterios de intervención terapéutica. Rev Española Obes; 2007 marzo.

19. Comissão das Comunidades Européias. Livro verde: melhorar a saúde mental da população. Rumo a uma estratégia de saúde mental para a União Européia. Bruxelas; 2005.

20. Agencia Española de Seguridad Alimentaria y Nutrición. Estrategia para la nutrición, actrividad física y prevención de la obesidad. Barcelona: NAOS; 2005.

21. Comisión de las Comunidades Europeas. Liblo blanco. Juntos por la salud: un planteamiento estratégico para la UE (2008-2013). Bruxelas; 2007

22. Organización Mundial de la Salud. Estratégia sobre régimen alimentario, acitividad física y salud. Geneva: OMS; 2004

23. Arrastegui J. La estrategia europea sobre problemas de salud relacionados con la alimentación, el sobrepeso y la obesidad. ReDeco: Rev electrónica de Derecho del Consumo y de la Alimentación. 2007; 15. Disponible en <http:// aibadaredeco.googlepages.com>.

24. Poulain JP. Manger aujourd'hui. Attitudes, normes et pratiques. Toulouse: Éditions Privat; 2002.

25. Gard M, Wright J. The obesity epidemic. London: Routledge; 2006

26. Campos P. The obesity myth. New York: Gotham Books; 2004.

27. Alemany M. Mecanismes de control del pes corporal. Rev Reial Acad Med Catalunya. 2003; 18(2):48.

28. Saguy AC, Riley WC. Weighing both sides: morality, mortality, and framing contests over obesity. J Health Politics, Policy and Law. 2005; 30(5): 869-921.

29. Lang y Rayner G. Overcoming policy cacophony on obesity: an ecological public health framework for policymakers. Obes Rev. 2007; 8(Suppl 1): 165-81.

30. Luque E. La obesidad, más allá del consumidor: raíces estructuraless de los entornos alimentarios. In: Díaz C, Gómez C. Alimentación, consumo y salud. Barcelona: Fundació "La Caixa"; 2008.

31. Contreras J, Gracia M. La alimentación y sus circunstancias. Barcelona: Alimentaria Exhibitions; 2004

Recibido el: 24/9/2008 Aprobado el: 18/2/2009 